\title{
Utility of High-Resolution Ultrasonography by Using Acoustic Coupler in Dermatology
}

\author{
Kazumi Fujioka ${ }^{1 *}$, Akira Fujioka ${ }^{2}$, Masahiro Okada ${ }^{1}$ \\ ${ }^{1}$ Department of Radiology, Nihon University School of Medicine, Tokyo, Japan \\ ${ }^{2}$ Department of Dermatology, Fujioka Dermatological Clinic, Tokyo, Japan
}

*Corresponding author: Kazumi Fujioka, Department of Radiology, Nihon University School of Medicine, 30-1, Oyaguchi kamicho,

Itabashi-ku, Tokyo, Japan

\section{ARTICLE INFO}

Received: 幽 December 04, 2019

Published: December 10, 2019

Citation: Kazumi Fujioka, Akira Fujioka, Masahiro Okada. Utility of High-Resolution Ultrasonography by Using Acoustic Coupler in Dermatology. Biomed J Sci \& Tech Res 23(5)-2019. BJSTR. MS.ID.003953.

Keywords: Dermal involvement; Acoustic Coupler; Real-Time Tissue Elastography; Dermatological Ultrasound; Subcutaneous Angiolipoma

\section{ABSTRACT}

We have described the experience using the acoustic coupler for subcutaneous angiolipoma comprehensively diagnosed in the lower extremity. The feature of ultrasonography (US) image with the acoustic coupler is more clearly depicted, particularly in superficial or near-field location. The usefulness of this procedure has been demonstrated as stableness state, equipped condition, and satisfactory observation for vasculature status due to the low attenuation material as previously described. The author will also recommend the observation with acoustic coupler for dermatologic superficial lesion, especially, rough, unevenness part in hand, foot, and joint location without copious amount of gel. We will indicate that acoustic coupler is useful for the appropriate condition to depict the accurate imaging on Real-Time Tissue Elastography (RTE). The author will suggest that this procedure can contribute to the accurate US diagnosis for dermatological superficial lesion, especially dermal involvement without a generous amount of gel.

Abbreviations: US: Ultrasonographiy; DERMUS: Dermatologic Ultrasound; RSS: Rodnan Skin Score; SWE: Share Wave Elastography; ECM: Extracellular Matrix; lcSSc: Limited Cutaneous Systemic Sclerosis; DT: Dermal Thickness; dcSSc: Diffuse Cutaneous Systemic Sclerosis; mRSS: Modified Rodnan Skin Score

\section{Introduction}

Dermatologic ultrasound imaging has been rapidly growing in recently years [1,2]. In currently dermatologic field, several reports focusing on ultrasonographic (US) features of the dermal involvement in systemic sclerosis (SSc) [3-5] have been provided. In addition, intradermal nodular fasciitis, primality dermis-seated [6-8] and angioleiomyoma, located close to or in contact with the dermis $[9,10]$ have been also reported. The author will indicate that the accurate US diagnosis for dermatological superficial lesions, particularly dermal involvement is significant. Well, acoustic coupler, namely SF-001 for superficial or near-field observation has been developed [11]. The usefulness of the acoustic coupler, SF-001 has been reported in the joint US of rheumatoid arthritis [11]. We have experienced the use of acoustic coupler for the dermatologic lesion for subcutaneous angiolipoma comprehensively diagnosed in the lower extremity. We have described the utilities of this procedure along with future perspective in dermatological ultrasound.

\section{Dermatological Ultrasound and Acoustic coupler}

The minimum frequency recommended for performing dermatologic examinations by DERMUS (Dermatologic Ultrasound) which is the international working group [1,2] was $15 \mathrm{MHz}$ [2]. We usually perform US studies for dermatologic lesions with a high-resolution, broad-band $(5 \mathrm{MHz}-18 \mathrm{MHz})$ linear transducer (Nobulus Hitachi, Ltd. Tokyo, Japan). We have also reported several studies of the dermatologic field [6-9, 12-18]. Carra et al. [19] technically suggested that only light pressure should be used to avoid compressing small vessels and missing flow. They prefer use of a copious amount of gel rather than a standoff pad. Shin et al. [20] mentioned that minimal transducer pressure was exerted 
while maintaining good contact and using a generous amount of gel to avoid underestimation of the blood flow on Doppler study. In current dermatology, Sulli et al.[3] strongly indicated that subclinical dermal involvement is detectable by high-resolution ultrasound even in skin areas with a normal modified Rodnan skin score (mRRS) in patients with limited cutaneous systemic sclerosis (SSc). It has been indicated that the most accurate US features of lesion activity are subcutaneous tissue echogenicity and cutaneous blood flow $[4,5]$. In addition, tumors of dermatological superficial location, namely intradermal nodular fasciitis [6-8], angioleiomyoma $[9,10]$, and primarily in dermis-seated tumors $[21,22]$ have been reported. Well, acoustic coupler, namely SF-001 for superficial or near-field observation has been developed [11]. We have previously described the experience using the acoustic coupler in the dermatologic lesion for panniculitis and superficial lipoma [18]. The author has recommended the observation for dermatologic superficial lesion, especially, rough, unevenness part in hand, foot, and joint location [18]. We also describe the utility of high-resolution ultrasonography using acoustic coupler for subcutaneous angiolipoma comprehensively diagnosed in the lower extremity. The author will propose that this procedure can contribute to the diagnostic accuracy of US for dermatological superficial lesion, especially dermal involvement without a copious amount of gel.

\section{Normal Skin Elasticity}

In the human dermis, cutaneous aging involves intrinsic and extrinsic agents. Cutaneous intrinsic aging is mainly attributed to dermal atrophy due to collagen loss, degeneration of the elastic fiber network, and loss of hydration [23,24]. Yang et al. [24] studied a reference range of normal skin elasticity evaluated by share wave elastography (SWE) in healthy subjects to interpret results in patients with systemic scleroderma. They concluded that skin elastic modules values estimated by SWE in healthy individuals are affected mainly by the skin site, sex, and age [24]. In addition, skin elasticity was higher in men than in women at each site and was increased in subjects aged 20 to 50 years than in the other age groups at the finger [24].

\section{Dermatologic Ultrasound Anatomy}

The US features of healthy skin is a layer of variable thickness depending on the site, the age and constitutional habitus of the patient [25]. The epidermis appears as a hyperechoic line in nonglabrous skin, while it is shown as bilaminar hyperechoic and parallel lines in glabrous skin. The dermis appears as a hyperechoic band, usually less bright than the epidermis, whereas subcutaneous fat layer appears as a hypoechoic with hyperechoic fibrous septa in between [4]. Wortsman [26] also suggested that the upper dermis in adults may show the subepidermal low-echogenicity band caused by deposits of glycosaminoglycans produced by photoaging. More deeply, the superficial fascia covering the muscular tissues can be seen as a hyperechoic regular line [27].

\section{Subcutaneous Angiolipoma}

Angiolipoma is a benign soft tissue tumor that was established in 1960 by Howard and Helwig [28]. Angiolipoma is common lipoma variants that account for $5 \%$ to $17 \%$ of all lipomas. Angiolipma usually indicates the noninfiltrating form affecting the subcutaneous layer [20]. They are also regarded as reactive or hamartomatous lesions because they typically have a normal karyotype [20,29]. Angiolipma can be painful and are one of the varieties of lipomas. Fibrin thrombi may be found within capillaries, and the degree of vascular proliferation may or may not be associated with pain [15, 30]. Motswaledi HM [31] suggested that angiolipoma may be painful, and the degree of pain varies with the degree of vascularization. The author has previously noted that angiolipoma is considered as one of the painful cutaneous and subcutaneous tumors accompanied with vascularized appearance using high-resolution ultrasound in dermatology [15]. Angiolipoma also tends to be smaller than $20 \mathrm{~mm}$ [20]. Choong et al. [32] reported that subcutaneous angiolipoma represented well-defined, predominantly hyperechoic masses containing small, patchy, hypoechoic areas on gray-scale US and showed a sparse internal vasculature on color Doppler US. Bang et al. [29] reported that most subcutaneous angiolipomas are oval-shaped, have well-defined margins, and hyperechoic appearance on gray-scale US. They also described that color Doppler US findings may help in differentiating angiolipoma from ordinary subcutaneous lipoma. According to Shin's report, angiolipoma appears as heterogeneous, hyperechoic, and ovoid shape with lesser visualized lateral tumor capsules on gray-scale US, while superficial lipoma appears as homogeneous, isoechoic, and spindle shape with well-visualized lateral capsules. They indicated that the differences were statistically significant [20]. In addition to these appearances, with respect to the presence of vascularity, tumor length, and tumor width, the difference was detected with statistical significance [20].

Furthermore, they noted that the sonographic-pathologic correlation demonstrated the statistically significant correlation between the tumoral vascularity and echogenicity. Figure 1 shows subcutaneous angiolipoma comprehensively diagnosed in the lower extremity location in a 75-year-old woman. Gray-scale US features represent well-defined, hyperechoic, and homogeneous with a small hypoechoic area. Tumor depth is $4.9 \mathrm{~mm}$, while tumor width is 5.8 $\mathrm{mm}$, showing smaller than $20 \mathrm{~mm}$ (Figure 1a). These appearances on gray-scale US are suggestive of subcutaneous angiolipoma. On RTE, red is used to represent high elasticity, blue represents low elasticity, and yellow or green shows intermediate elasticity [33]. Klauser et al. suggested that sonoelastography requires a modest distance, typically $3 \mathrm{~mm}$, between the probe surface and the tissues to be evaluated [33,34]. Thus, when operator examine the superficial lesion, stand-off pads are useful to increase the distance between the probe and the area of interest [33,34]. Park et al. [35] also described that the strain elastography score was classified into four grades (1-4) according to their elasticity; score 1 (very 
soft, high elasticity), score 2 (moderately soft, moderately high elasticity), score 3 (moderately hard, moderately low elasticity), and score 4 (very hard, low elasticity). The dermis consisting of connective tissue, nerves, blood, and lymphatic vessels, gland, mast cells, fibroblasts, histiocytes [27] represents blue color, showing hard nature, namely, low elasticity. While the subcutaneous fat tissue shows red color, consistent with soft nature, suggesting high elasticity, and reflecting contents of adipose cells [27] on RTE. The nodule represents blue color with a green color, showing moderately hard nature, consistent with moderately low elasticity (Figure 1b). Color Doppler US revealed no substantial blood flow signals in the nodule (Figure 2). The US imagings were depicted more clearly, especially in superficial or near-field location equipped with the acoustic coupler (SF-001) due to the stableness state, equipped condition, and low attenuation material status. With respect to RTE, it is putative that this procedure can contribute to the appropriate condition to depict the accurate US features.

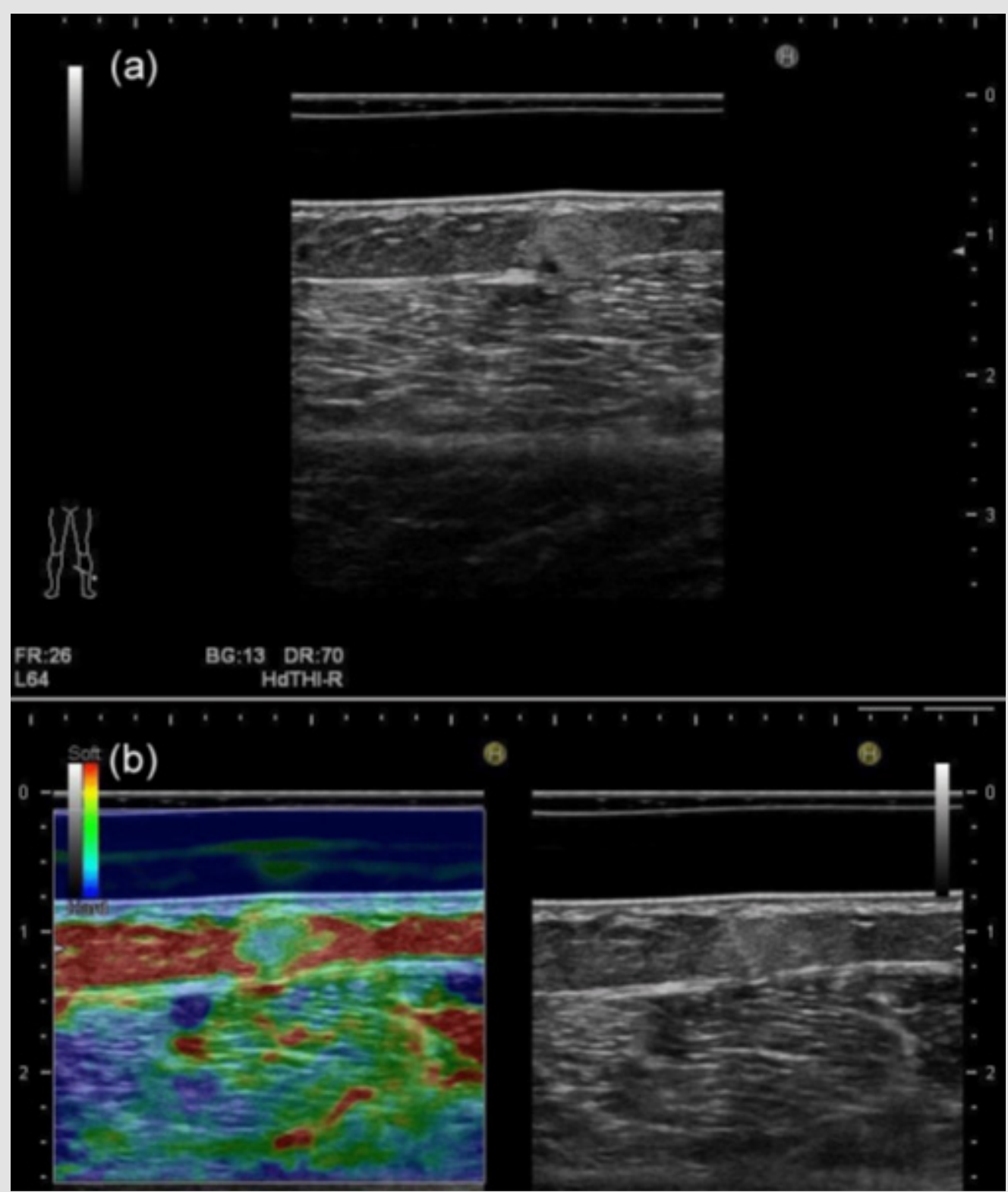

Figure 1: Subcutaneous angiolipoma comprehensively diagnosed in the lower extremity location in a 75-year-old woman.

a) The nodule shows well-defined hyperechoic, and homogeneous with small internal hypoechoic area, suggesting subcutaneous angiolipoma on gray-scale US.

b) The dermis represents blue color, showing hard nature, namely, low elasticity, while the subcutaneous fat tissue shows red color, consistent with soft nature, suggesting high elasticity on Real-Time Tissue Elastography. The nodule represents blue color with a green color, showing moderately hard nature, consistent with moderately low elasticity. 


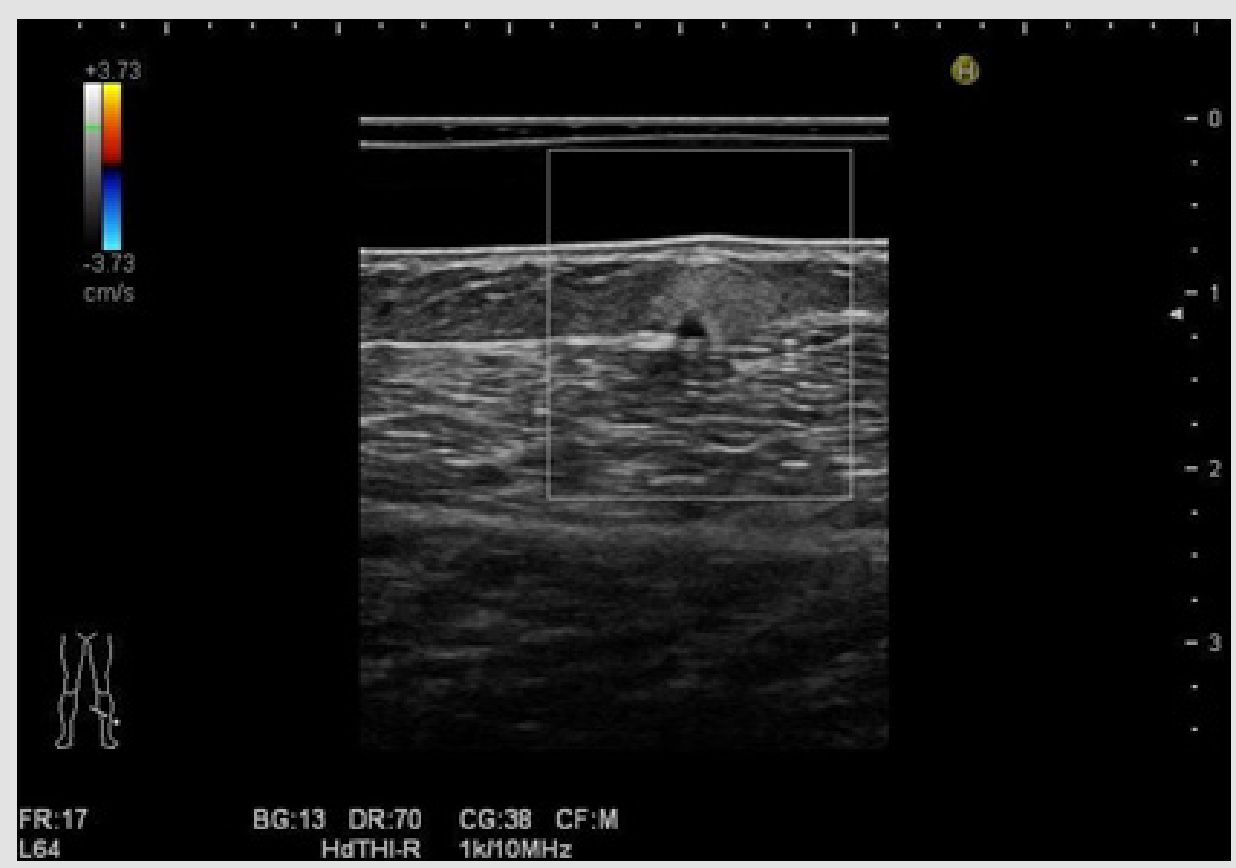

Figure 2: Blood flow signals were not shown in the nodule on color Doppler US.

\section{Future Viewpoint in Dermatological Ultrasound}

Systemic sclerosis (SSc, scleroderma) is characterized by fibrosis in skin and internal organs. The fibrotic process involves cytokines, such as TGF- $\beta$, that are released by a variety of immune cells, continuously stimulating fibroblast production of extracellular matrix (ECM). The established method of skin assessment in the semi-quantitative scoring (Rodnan skin score, Rss), introduced by Rodnan in 1979 [36,37]. Fibroblasts from patients with more rapid changes in skin thickness and skin echogenicity as assessed by ultrasound have been shown to produce more ECM in vitro. Low echogenicity and high thickness on gray-scale US represent edema, showing the increased ECM production, and suggesting early or aggressive skin disease $[36,38]$. The modified Rodnan skin score (mRSS), the validated method used to assess the severity of skin thickening in SSc, and to distinguish between patients with either limited cutaneous systemic sclerosis (lcSSc) or diffuse cutaneous systemic sclerosis (dcSSc) [3]. Sulli et al. [3] have suggested that subclinical dermal involvement may be detectable by US even in skin areas with a normal mRSS in patients with lcSSC. Statistically, significant higher mean dermal thickness (DT) was recognized in almost all skin areas in comparison with healthy subjects has been indicated [3].The US features of systemic sclerosis can vary through the different phases of the disease, showing from thickening and decreased echogenicity of the dermis and areas with increased echogenicity of the subcutaneous tissue during the active phase to substantial atrophy of the dermis and subcutaneous tissue during the late phase. It has been suggested that the most accurate US features of the activity of this entity are increased subcutaneous tissue echogenicity and cutaneous blood flow [4,5,39]. The author will propose that this procedure can contribute to the accurate US diagnosis for dermal involvement, namely dermal thickness, echogenicity, vasculature condition in SSc.

\section{Conclusion}

1) We have described the experience using the acoustic coupler for comprehensively diagnosed subcutaneous angiolipoma.

2) It is putative that this procedure with acoustic coupler is useful for the appropriate condition to depict the accurate imaging on Real-Time Tissue Elastography.

3) The author will suggest that this procedure can contribute to the accurate ultrasonographic diagnosis for dermatological superficial lesion, especially dermal involvement, namely thickness, echogenicity, vasculature condition without a copious amount of gel.

\section{Acknowledgement}

The author deeply appreciates Dr. Minoru Oishi for his kind support.

\section{References}

1. Wortsman X, Alfageme F, Roustan G, Arias Santiago S, Martorell A, et al. (2016) Guidelines for performing dermatologic ultrasound examinations by the DERMUS group. J Ultrasound Med 35(3): 577-580.

2. Wortsman X, Alfageme F, Roustan G, Arias-Santiago S, Martorell A, et al. (2016) Proposal for an assessment training program in dermatologic ultrasound by the DERMUS group. J Ultrasound Med 35(11): 2305-2309.

3. Sulli A, Ruaro B, Smith V, Paolino S, Pizzorni C, et al. (2017) Subclinical dermal involvements is detectable by high frequency ultrasound even in patients with limited cutaneous systemic sclerosis. Arthritis Res Ther 19: 61. 
4. Wortsman X (2012) Common applications of dermatologic sonography J Ultrasound Med 31(1): 97-111.

5. Wortsman X, Wortsman J, Sazunic I, Carreno L (2011) Activity assessment in morphea using color Doppler ultrasound. J Am Aad Dermatol 65(5): 942-948.

6. Fujioka K, Fujioka A, Oishi M,Eto H, Tajima S, etal. (2017) Ultrasonography findings of intradermal nodular fasciitis a rare case report and review of the literature. Clin Exp Dermatol 42(3): 335-336.

7. Fujioka K, Fujioka A, Tajima S, Oishi M, Hayashi K, et al. (2018) Characteristic power Doppler sonographic imaging of nodular fasciitis from a dermatological perspective: another case and review of three cases. J Clin Case Rep 8(9): 1165.

8. Fujioka K (2019) Characteristic appearances of nodular fasciitis on high-resolution ultrasonography: with vasculature status from a lesionlocated perspective. Biomed J Sci \& Tec Res 20(4).

9. Fujioka K, Fujioka A, Oishi M, Hayashi K, Nakayama T (2018) Highresolution ultrasound imaging for angioleiomyoma: a painful and vascularized superficial tumor. Biomed J Sci \& Tec Res 9(5).

10. Zhang JZ, Zhou J, Zhang ZC (2016) Subcutaneous angioleiomyoma: clinical and sonographic features with histopathologic correlation. Ultrasound Med 35(8): 1669-1673.

11. Sakamoto F, Narita A, Henmi M, Asano A, Tanimura K, et al. (2017) Evaluation of new acoustic coupler: SF-001 in the rheumatic ultrasound. MEDIX 67: 12-16.

12. Fujioka K, Fujioka A, Eto H, Suzuki K, Sanuki E, et al. (2006) Nodular fascitis in the thigh followed up using ultrasonography. J Med Ultrason 33: 49-53.

13. Fujioka K (2018) Presentations of clinical ultrasonographic and pathological features of nodular fasciitis from an established cytogenetic viewpoint: review of the case series. J Carcinog Mutagen 9(4): 326.

14. Fujioka K (2018) Usefulness of comprehensive high-resolution ultrasound imaging in dermatologic field: epidermal cyst. Biomed J Sci \& Tec Res 12(1)

15. Fujioka K (2019) Painful cutaneous and subcutaneous tumors accompanied with vascularized appearance using high-resolution ultrasound in dermatology: The acronym "ENGLAND" or "LEND AN EGG". Biomed J Sci \& Tec Res 12(4)

16. Fujioka K (2019) Real- time Tissue elastography in epidermal cyst: along with enhanced MRI and elasticity features of peripheral rim. Biomed J Sci \& Tec Res 16(5).

17. Fujioka K (2019) A comparison between superficial and deep-seated lipomas on high-resolution ultrasonography: with RTE and MRI appearances. Biomed J Sci \& Tec Res 19(2).

18. Fujioka K, Fujioka A, Oishi M, Masahiro Okada (2019) A new application in dermatological ultrasound. Biomed J Sci \& Tec Res 22(5)

19. Carra BJ, Bui-Mansfield LT, O’Brien SD, Chen DC (2014) Sonography of musculoskeletal soft-tissue masses: techniques, pearls, and pitfalls. AJR Am J Roentgenol 202(6): 1281-1290.

20. Shin YS, Kim YJ, Park IS, Chu YC, Kim JH, et al. (2016) Sonographic differentiation between angiolipomas and superficial lipomas. J Ultrasound Med 35(11): 2421-2429.

21. Jin W, Kim GY, Park SY, Chun YS, Nam DH, et al. (2010) The spectrum of vascularized superficial soft-tissue tumors on sonography with a histopathologic correlation: part 1, benign tumors. AJR Am J Roentgenol 2195: 439-445

22. Hwang CM, Kang BS, Hong HJ, LeeJY, Suh JH, et al. (2018) Ultrasonographic features of eccrine spiradenoma. J Ultrasound Med 37(5): 1267-1272.

23. Scotto di Santolo M, Saqnelli M, Mancini M, Scalvenzi M, Delfino M, et al. (2015) High-resolution color-Doppler ultrasound for the study of skin growths. Arch Dermatol Res 307(7): 559-566.

24. Yang Y, Wang L, Yan F, Xiang X, Tang Y, et al. (2018) Determination of normal skin elasticity by using real-time share wave elastography. J Ultrasound Med. 37(11): 2507-2516.

25. Crisan D, Lupsor M, Boca A, Crisan M Badea R, et al. (2012) Ultrasonographic assessment of skin structure according to age. Indian J Dermatol Venereol Leprol 78(4): 519.

26. Wortsman X (2017) Sonography of dermatologic emergencies. J Ultrasound Med 36(9): 1905-1914.

27. Mandava A, Ravuri PR, Konathan R (2013) High resolution ultrasound imaging of cutaneous lesions. Indian J Radiol Imaging 23(3): 269-277.

28. Howard WR, Helwig EB (1960) Angiolipoma. Arch Dermatol 82: 924931.

29. Bang M, Kang BS, Hwang JC, Weon YC, Choi SH, et al. (2012) Ultrasonographic analysis of subcutaneous angiolipoma. Skeletal Radiol 41(9): 1055-1059.

30. Naversen DN, Trask DM, Watson FH, Burket JM (1993) Painful tumors of the skin: "LEND AN EGG”. J Am Acad Dermatol 28: 298-300.

31. Motswaledi HM (2010) Multiple nodules on the trunk and arms. Int J Dermatol 49:1349-1350.

32. Choong KK (2004) Snographic appearances of subcutaneous angiolipomas. J Ultrasound Med 23: 715-717.

33. Klauser AS, Miyamoto H, Bellmann-Weiler R, Feuchtner GM, Wick MC et al. (2014) Sonoelastography: musculoskeletal applications. Radiology 272(3): 622-633.

34. Klauser AS, Faschingbauer R, Jaschke WR (2010) Is sonoelastography of value in assessing tendons? Semin Musculoskelet Radiol 14(3): 323333.

35. Park HJ, Lee SY, Lee SM, Kim WT, Lee S, et al. (2015) Strain eastography features of epidermoid tumours in superficial soft-issue: differences from other benign soft tissue tumours and malignant tumours. Br J Radiol 88 (1050): 20140797.

36. Hesselstrand R, Scheja A, Wildt M, Akesson A (2008) High frequency ultrasound of skin involvement in systemic sclerosis reflects oedema, extension and severity in early disease. Rheumatology 47(1): 84-87.

37. Rodnan GP, Lipinski E, Luksick J (1979) Skin thickness and collagen content in progressive systemic sclerosis and localized scleroderma. Arthritis Rheum. 22(2): 130-140.

38. Hesselstrand R, Westergren-Thorsson G, Scheja A, Wildt M, Akesson A, et al. (2002) The association between changes in skin echogenicity and the fibroblast production of biglycan and versican in systemic sclerosis. Clin Exp Rheumatol 20(3): 301-308.

39. Li SC, Liebling MS, Haines KA (2007) Ultrasonography is a sensitive tool for monitoring localized scleroderma. Rheumatology 46(8): 1316-1319. 
ISSN: 2574-1241

DOI: 10.26717/BJSTR.2019.23.003953

Kazumi Fujioka. Biomed J Sci \& Tech Res

(C) This work is licensed under Creative

Submission Link: https://biomedres.us/submit-manuscript.php

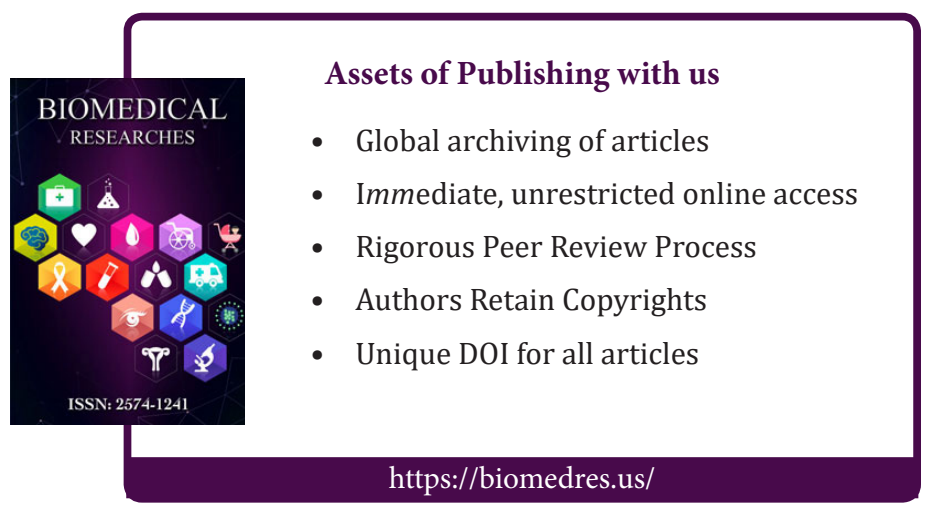

